\title{
A virosomal formulated Her-2/neu multi-peptide vaccine induces Her-2/neu-specific immune responses in patients with metastatic breast cancer: a phase I study
}

\author{
Ursula Wiedermann • C. Wiltschke $\cdot$ J. Jasinska • \\ M. Kundi - R. Zurbriggen - E. Garner-Spitzer · R. Bartsch • \\ G. Steger $\cdot$ H. Pehamberger $\cdot$ O. Scheiner $\cdot$ C. C. Zielinski
}

Received: 17 July 2009/Accepted: 21 November 2009/Published online: 11 December 2009

(C) Springer Science+Business Media, LLC. 2009

\begin{abstract}
We have previously shown in mice that vaccination with three Her-2-peptides representing B-cell epitopes of the extracellular domain of Her-2/neu induces Her-2/neu-specific IgG antibodies with strong anti-tumor activity in vitro and in vivo. We have now finalized a phase I clinical trial with an anti-Her-2/neu vaccine-construct of immunopotentiating reconstituted influenza virosomes with the three peptides in patients with metastatic breast cancer $(\mathrm{MBC})$. Ten MBC patients with low protein overexpression of Her-2/neu of MBC (+ or ++ upon immunohistochemistry, FISH negative) and positive hormone receptor status were enrolled in a single center phase I study. The
\end{abstract}

U. Wiedermann and C. Wiltschke contributed equally to this work.

U. Wiedermann $(\bowtie) \cdot$ J. Jasinska · E. Garner-Spitzer .

O. Scheiner

Institute of Specific Prophylaxis and Tropical Medicine,

Medical University of Vienna, Kinderspitalgasse 15,

1090 Vienna, Austria

e-mail: ursula.wiedermann@meduniwien.ac.at

C. Wiltschke $\cdot$ R. Bartsch - G. Steger - C. C. Zielinski

Clinical Division of Oncology, Department of Medicine I,

Medical University of Vienna, Vienna, Austria

M. Kundi

Department of Environmental Medicine, Medical

University of Vienna, Vienna, Austria

R. Zurbriggen

Pevion Biotech LTD, Bern, Switzerland

H. Pehamberger

Department of Dermatology, Medical University of Vienna,

Vienna, Austria

J. Jasinska · H. Pehamberger - O. Scheiner - C. C. Zielinski

BioLife Science, Vienna, Austria virosomal formulated vaccine, consisting of $10 \mu \mathrm{g} /$ peptide, was intramuscularly applied three times on days 1,28 , and 56. The primary endpoint of the study, which lasted 12 weeks, was safety, the secondary endpoint immunogenicity. Local erythema at the injection site was the only vaccine-related side effect occurring in four patients. In 8 of 10 patients an increase in peptide-specific antibody titer measured by ELISA was found. Importantly, the induced antibodies were also directed against the native Her-2/neu protein. Cellular immune responses, as measured by in vitro production of IL-2, IFN- $\gamma$, and TNF- $\alpha$ of PBMCs showed a marked increase after vaccination in the majority of vaccinees. Notably, the number of CD4+CD25+Foxp3+T regulatory cells, which were significantly increased compared to healthy controls prior to vaccination, was markedly reduced following vaccination. In all, the immunological responses after vaccination indicated that the patients in stage IV of disease were immunocompetent and susceptible to vaccination. The Her-2/neu multipeptide vaccine was safe, well tolerated and effective in overcoming immunological tolerance to Her-2/neu. The induction of anti-Her-2specific antibodies could result in clinical benefit comparable to passive anti-Her-2 antibody therapy.

Keywords Her-2/neu - B-cell peptides · Vaccination . Virosomes $\cdot$ Antibodies $\cdot \mathrm{T}$ cell $\cdot \mathrm{T}$ regulatory cells . Phase I trial

\section{Introduction}

The Her-2/neu protein is overexpressed in $15-20 \%$ of breast cancers. Its primarily detrimental prognostic potential including rapid progression from early to metastatic breast cancer, the formation of metastases of mainly 
visceral origin and rapid disease progression to death $[1,2]$ has been reversed by the introduction of trastuzumab in the late 1990's and the first decade of the current century. Trastuzumab is a monoclonal antibody directed against the extracellular domain of the Her-2/neu protein. Its clinical use has resulted not only in significant prolongation of disease-free and overall survival in early breast cancer, but also in the significant prolongation of overall survival in patients with metastatic breast cancer overexpressing the Her-2/neu protein. Trastuzumab toxicity mainly consists of the induction of congestive heart failure which occurs only in a very small percentage of patients [3].

The therapeutic administration of such monoclonal antibodies as trastuzumab, cetuximab, and rituximab targeting various proteins on the tumor cell membrane has brought about a milestone in the treatment of certain malignant diseases [4,5]. Although of high therapeutic efficacy, the passive antibody administration suffers from some drawbacks including the need of frequent administration of the drug in varying, but usually rather short time intervals, the necessity for a prolonged duration of drug delivery and the impossibility of application in a prophylactic manner in high-risk patients. All these difficulties could be circumvented by the generation of an active immune response aimed at the identical target induced by vaccination with an appropriate substance. However, clinical studies on the efficacy of various vaccines used in cancer patients have resulted in limited results due to cancer- or treatment-related anergy with low seroconversion rates, HLA-restriction of vaccine-induced cytotoxic $\mathrm{T}$ cells and, finally, inappropriate immunologic targeting $[6,7]$.

In the present article, we report on a phase I clinical study in patients with metastatic breast cancer, using a vaccine directed against three peptides derived from the extracellular domain of the Her-2/neu protein. These peptides were preselected by computer-aided prediction to search for B-cell epitopes able to induce anti-Her-2/neu antibody responses [8]. In previously published animal studies, we have shown that these peptides, when coupled to tetanus toxoid and used for immunization in mice, induced anti-Her-2/neu antibodies that were able to inhibit growth of Her-2/neu overexpressing SKBR-3 cells in vitro via direct proliferation inhibition, antibody-dependent cellular cytotoxicity as well as complement-dependent cell lysis [8]. Using a $c-n e u$ transgenic mouse model we further demonstrated that immunization with this multi-epitope vaccine led to delayed tumor growth onset and reduced tumor growth progression in vivo [9]. To enhance the antigenicity against self-antigens for a human phase I study, we have chosen to couple these peptides to immunopotentiating reconstituted influenza virosomes (IRIV), which have been shown to exert impressive adjuvant characteristics in the context of vaccines directed at different targets associated with infectious diseases [10, 11, 12]. Here, we report on the primary endpoint of this phase I trial consisting of safety and tolerability of the used vaccine, but also on secondary endpoint which included immunogenicity of the multi-peptide vaccine. It is shown that the vaccine yielded a specific immune response in eight out of ten individuals with metastatic breast cancer and exerted only very mild, local side effects, thus making it a good candidate for further clinical phase II and phase III trials.

\section{Patients and methods}

Her-2/neu peptide-based vaccine

The peptide-based vaccine contains three immunogenic, putative B-cell peptides derived from the extracellular domain of Her-2/neu: P4 (378-394), P6 (545-560), and P7 (610-623) as previously reported [9]. Peptides were associated in a defined fashion to immunopotentiating reconstituted influenza virosomes (IRIV) [10]. These are spherical, unilamellar vesicles consisting of a mixture of natural and synthetic phospholipids and membrane glycoproteins. Native inactivated viruses were first dissolved in detergent and the viral nucleocapsids were removed. Her-2/ neu peptides coupled to phosphatidylethanolamine and hemagglutinin, originating from the influenza A/Singapore/ 86 strain, were then integrated into the virosome membrane during reconstitution process. The final vaccine formulation (PEV 6) contained $10 \mu \mathrm{g}$ of each of the three peptides incorporated into the virosomes. The vaccine was manufactured by Pevion Biotech Ltd (Bern, Switzerland) according to the rules of Good Manufacturing Practices including tests for sterility, pyrogenicity, and stability. The vaccine was supplied in 1-ml ready to use syringes for each vaccination.

\section{Patients}

Patients with metastatic breast cancer were eligible for the study. Measurable disease was not required as study inclusion criteria, because metastatic breast cancer patients with bone only disease were also included in the study. These patients had to present elevated tumor markers (CA 15-3), and levels of tumor markers were used for response evaluation.

Furthermore, patients were required to have positive hormone receptor (estrogen and/or progesterone receptor) and low Her-2/neu overexpression ( + or ++ upon immunohistochemistry, IHC, by HercepTest; DAKO, Carpinteria, $\mathrm{CA}$ - and negative by fluorescence in situ hybridization, FISH). Previously administered chemotherapy was allowed, 
Table 1 Patients'

characteristics are listed with respect to age, onset of disease, Her-2/neu expression, prior chemotherapy, and current hormone treatment

\begin{tabular}{llllll}
\hline $\begin{array}{l}\text { Pat. } \\
\text { number }\end{array}$ & $\begin{array}{l}\text { Age } \\
\text { (years) }\end{array}$ & $\begin{array}{l}\text { Met. disease } \\
\text { since }\end{array}$ & $\begin{array}{l}\text { Her-2 } \\
\text { status }\end{array}$ & Prior chemotherapy & $\begin{array}{l}\text { Current antihorm. } \\
\text { therapy }\end{array}$ \\
\hline 1 & $55 \mathrm{a}$ & Oct. 2006 & ++ & No & Anastrozol \\
2 & $66 \mathrm{a}$ & May 2004 & + & Yes (1 adj) & Fulvestrant \\
3 & $84 \mathrm{a}$ & Mar. 1999 & ++ & No & Anastrozol \\
4 & $79 \mathrm{a}$ & Sept. 2003 & ++ & No & Anastrozol \\
5 & $67 \mathrm{a}$ & Apr. 2004 & ++ & No & Fulvestrant \\
6 & $69 \mathrm{a}$ & Sept. 2004 & + & No & Anastrozol \\
7 & $60 \mathrm{a}$ & Aug. 2002 & + & Yes (3 met) & Fulvestrant \\
8 & $76 \mathrm{a}$ & Apr. 1999 & + & No & Fulvestrant \\
9 & $63 \mathrm{a}$ & Jun. 2006 & + & Yes (1 met) & Exemestan \\
10 & $70 \mathrm{a}$ & Apr. 2008 & + & NO & Anastrozol \\
\hline
\end{tabular}

but no concomitant chemotherapy was permitted for inclusion of patients into the study protocol. All patients received endocrine therapy (tamoxifen or aromatase inhibitors) for treatment of their disease. Patients with positive results of Her-2/neu testing upon IHC $(+++)$ or FISH were excluded. Ten patients were enrolled in the study. Characteristics of the patients are shown in Table 1.

\section{Study design}

We report on a single center, non-randomized phase I study. Primary endpoint was to demonstrate the safety and tolerability of the virosomal formulated Her-2/neu multipeptide vaccine. Secondary endpoint was to determine the immune response against the three virosomal formulated Her-2/neu-derived peptides. The vaccine was the virosomal formulation of $10 \mu \mathrm{g} /$ peptide administered in patients intramuscularly on days 1,28 , and 56 . The total duration of the study for each patient was 12 weeks. This phase I study was approved by the Ethical Review Board of the Medical University Vienna and the General Hospital Vienna and was monitored by four independent outside reviewers who were assembled as an independent data safety monitoring board (DSMB).

\section{Safety assessments}

Clinical examination and laboratory assessment of blood counts, clinical chemistry, and liver function were carried out at screening, before each of the three vaccinations, at final assessment, and ad hoc as clinically indicated. Left ventricular ejection fraction (LVEF) was assessed by echocardiography at screening and final assessment. Assessment of health status, including local vaccination reactions were furthermore performed 2 and 7 days after each vaccination. Adverse events were assessed every cycle for the duration of the trial and graded according to the National Cancer Institute Common Toxicity Criteria (NCI CTC), version 2.0. Data on serious adverse events (SAEs) were collected throughout the study.

Evaluation of response

Although not defined as a goal in this phase I study, patients had to undergo CT scans as part of their routine clinical observance. CT scans were performed routinely every three months or earlier in the case of a change in the clinical course of the disease. Responses were defined according to the Response Evaluation Criteria in Solid Tumors (RECIST).

Sample collection for immunological assays

Blood samples $(40 \mathrm{ml})$ were taken prior to vaccination and 4 weeks after the last immunization. Serum was stored at $20^{\circ} \mathrm{C}$ until analysis. Sera from healthy volunteers $(n=16)$ were used for background determination in ELISA.

PBMC were separated on Ficoll-Paque density gradients as described elsewhere [13] and cryopreserved until evaluation. In addition, PBMC were isolated from eight ageand gender-matched healthy volunteers prior and after vaccination with a hepatitis A vaccine (HAVRIX 1440) [13].

\section{Peptide-specific responses}

Ninety-six-well microtiter plates (Nunc-Immuno Plate, Nalge Nunc International, Denmark) were coated with $5 \mu \mathrm{g} / \mathrm{ml} \mathrm{Her-2/neu} \mathrm{peptides} \mathrm{conjugated} \mathrm{to} \mathrm{KLH} \mathrm{(synthes-}$ ised and coupled by PiChem, Austria) or $5 \mu \mathrm{g} / \mathrm{ml} \mathrm{KLH}$ (SIGMA, St. Louis, MO, USA) in $100 \mathrm{mM}$ carbonate buffer, pH 9.6 overnight at $4^{\circ} \mathrm{C}$. Plates were washed with PBS and non-specific binding sites were blocked for $5 \mathrm{~h}$ with PBS containing 2\% milk powder (Roth-Lactan, Austria). Serial dilutions of sera 1:100, 1:200, 1:400, 1:800, 
$1: 1,600$, and 1:3,200 were prepared in PBS-tw $(0.05 \% \mathrm{v} / \mathrm{v}$ Tween 20) containing $0.5 \%$ milk powder and incubated overnight at $4^{\circ} \mathrm{C}$. After washing with PBS-tw, HRP-labeled anti-human IgG (SIGMA, St. Louis, MO, USA) diluted 1:60,000 in PBS-tw containing $0.5 \%$ milk powder were added to the plates and incubated for $2 \mathrm{~h}$ at room temperature. Color development was performed with tetramethylbenzidin (TMB) substrate (Chemicon, Temecula, CA, USA) and was stopped with $\mathrm{H}_{2} \mathrm{SO}_{4}$ after 10 min. Absorbance was measured with TECAN Genios reader. The resulting $\mathrm{OD}$ of each serum dilution was calculated by subtracting the OD obtained on KLH-coated wells from the OD values of the peptide-KLH coated wells. Assay control was performed with defined patient's serum and defined negative serum pool.

Titer was defined as serum dilution to obtain an $\mathrm{OD}=1.0$ over the background derived from healthy donors. Pre- and post-vaccination titers were calculated.

\section{Her-2/neu-specific responses}

Recombinant human ErbB2/Fc Chimera (R\&D Systems, MN, USA) consisting of the extracellular domain, sixamino acid linkage, and the human IgG1 Fc fragment was used as coating antigen. Plates were coated with $1 \mu \mathrm{g} / \mathrm{ml}$ ErbB2/Fc Chimera in carbonate buffer overnight at $4^{\circ} \mathrm{C}$. Control plates were coated with the analogous dilution of the reconstitution buffer for ErbB-2-Fc Chimera containing $0.1 \%$ BSA. Plates were blocked with $5 \%$ milk powder in PBS for $5 \mathrm{~h}$ before serial dilutions of sera as listed above were added and incubated overnight at $4{ }^{\circ} \mathrm{C}$. Bound $\operatorname{Ig} \kappa$ and Ig $\lambda$ antibodies were detected with HRP-labeled anti-human $\kappa$ and anti $\lambda$ chain IgG (SIGMA, St. Louis, MO, USA), respectively, diluted 1:10,000 in PBS-tw/0.5\% milk. Herceptin $^{\circledR}$ (Roche, Switzerland) titration curve 0.05$0.003125 \mu \mathrm{g} / \mathrm{ml}$ was used as control in assays with $\operatorname{Ig} \kappa$ detection and serum obtained from patient with Her-2/ neu +++ positive tumor for the $\operatorname{Ig} \lambda$ detection. ELISA was developed with TMB substrate and stopped after $20 \mathrm{~min}$. Titer was calculated as described above. The ratio postvaccine titer/pre-vaccine titer represents titer increase. Ratio greater than 1 was regarded as Her-2/neu-specific titer increase.

\section{Western blot}

SK-BR-3 cells were lysed as described elsewhere [8]. The protein content was quantified with BCA Protein assay kit (Thermo scientific Rockford, IL USA). Her-2/neu was immunoprecipitated from a total of $16 \mathrm{mg}$ protein with $200 \mu \mathrm{g} \mathrm{Herceptin}{ }^{\circledR}$ (Roche, Basel, Switzerland) and $150 \mu \mathrm{l}$ protein $\mathrm{A}+\mathrm{G}$ agarose (Merck-Calbiochem, Darmstadt, Germany) in $0.1 \mathrm{M}$ TBS over night at $4^{\circ} \mathrm{C}$. Pellet was washed twice in $0.1 \mathrm{M}$ TBS $\mathrm{pH} 7.4$ and twice in $0.1 \mathrm{M}$ TBS with $0.5 \%$ Nonidet P40. Precipitate was separated on 8\% SDS PAGE and blotted on nitrocellulose membranes (Schleicher \& Schuell, Dassel, Germany). Strips were blocked with $2 \%$ milk in $0.1 \mathrm{M}$ TBS buffer and then incubated over night with sera diluted 1:25 in 0.1 M TBS$0.05 \% \mathrm{v} / \mathrm{v}$ Tween-20 containing $0.5 \%$ milk. Rabbit antiHer-2/neu antibody (Zymed, San Francisco, CA, USA) was used as positive control and serum obtained from healthy volunteers were as negative controls. After three washing steps, bound human IgG was detected by AP-labeled antihuman IgG (SIGMA, Saint Louis, Missouri, USA) and subsequently stained with NBT/BCIP substrate mixture. Rabbit IgG was detected by AP-swine anti-rabbit antibody (DAKO A7S Denmark).

Hemagglutination-inhibition assay

The analysis of the serum samples for neutralizing antibody levels against influenza were performed according to standard methodology [14]. Two serial twofold dilutions of the sera were prepared. The diluted sera were incubated with either the hemagglutinin antigen of the Influenza strain A/Sing/86 or with the solvent. Thereafter a suspension of chicken erythrocytes was added. Hemagglutination occurred with virus antigen in the absence of sufficient inhibiting antibodies. The hemagglutination inhibition titer is the reciprocal value of the highest dilution which inhibits hemagglutination. The titer assigned to each sample is the geometric mean of two independent determinations (EMEA criteria in Appendix 13.3). A fourfold increase $(\geq 4)$ in the titers after vaccination was taken as positive vaccine response.

\section{Cellular responses specific to virosomal antigens}

Peripheral blood $\mathrm{T}$ cell responses were assessed by cytokine production in vitro upon stimulation with virosomes. PBMC were cultured at $1 \times 10^{6} /$ well in medium (RPMI with $10 \%$ heat inactivated human $\mathrm{AB}$ serum) containing virosomes with total hemagglutinin content of $8 \mu \mathrm{g} / \mathrm{ml}$ or $1 \mu \mathrm{g} / \mathrm{ml}$ streptococcal enterotoxin B (SIGMA, Saint Louis, Missouri, USA) as positive control antigen and in the absence of any antigens (at least three wells per antigen). The virosome formulation was kindly provided by Pevion Biotech Ltd (Bern, Switzerland). The final culture volume was $200 \mu \mathrm{l} /$ well, culture conditions were $37^{\circ} \mathrm{C}$ and $5 \%$ $\mathrm{CO}_{2}$. Supernatants were harvested after $48 \mathrm{~h}$ and stored frozen until measurement of IFN- $\gamma, \mathrm{TNF}-\alpha$, and IL- 2 by ELISA.

Commercially available ELISA kits were used for measurement of TNF- $\alpha$ (R\&D, MN, USA) and IL-2 
(ENDOGENE/Thermo Scientific, IL, USA). Assays were carried out according to manufacturer's instructions.

For IFN- $\gamma$ levels determination plates were coated with $0.5 \mu \mathrm{g} / \mathrm{ml}$ mouse anti-human IFN- $\gamma$ (Calbiochem, NJ, USA) in carbonate buffer over night at $4^{\circ} \mathrm{C}$. After blocking with $4 \%$ BSA (bovine serum albumin) in PBS, $50 \mu \mathrm{l}$ of undiluted samples were added at room temperature for $1 \mathrm{~h}$. Recombinant human IFN- $\gamma$ (ENDOGENE/Thermo Scientific, IL, USA) in concentration ranging from 8 to $500 \mathrm{pg} /$ $\mathrm{ml}$ was used to generate a calibration curve. Plates were subsequently washed with PBS-tw. Biotin-labeled mouse anti-human IFN- $\gamma$ (Thermo Scientific, IL, USA) at a concentration $0.9 \mu \mathrm{g} / \mathrm{ml}$ was applied, followed by peroxidaseconjugated streptavidin (Thermo Scientific) 1:15,000 in PBS-tw/4\% BSA for $30 \mathrm{~min}$. The assay was developed with TMB substrate. Cytokine levels represent results after subtraction of unstimulated medium values.

\section{Flow cytometry}

Immunofluorescent staining of cell surface markers on PBMC was performed on patients and eight healthy ageand gender-matched volunteers. Cryopreserved cells were washed with $\mathrm{PBS} / 0.5 \% \mathrm{BSA} / 0.5 \%$ sodium azide, diluted to $5 \times 10^{5}$ cells per micronic tube, blocked with $20 \%$ human AB serum for $20 \mathrm{~min}$ and subsequently stained with directly conjugated $\mathrm{mAbs}$ at predetermined optimal concentrations for $30 \mathrm{~min}$ at $4{ }^{\circ} \mathrm{C}$ in the dark. Antibodies used: $\alpha$ CD3-PE-Cy5, $\alpha$ CD19-FITC (IgG1 k), $\alpha$ CD27-PE, $\alpha$ CD4FITC (IgG2 k), $\alpha$ CD4-PE-Cy5 (IgG1 k), $\alpha$ CD28-PE-Cy5 (IgG1 k), $\alpha$ CD8-APC (IgG1 k), $\alpha$ CD45R0-PE (IgG2a k), $\alpha$ CD62L-FITC (IgG1 k), and $\alpha$ CD25-PE (IgG1 k). All antibodies were purchased from BD Pharmingen, CA, USA. Antibody binding to PBMC was analyzed on a FACS CALIBUR flow cytometer (BD Biosciences) by gating on cells with forward and side light scatter properties of lymphocytes, using BD CellQuest software. Isotype matched negative control reagents were used to verify the staining specificity of the experimental antibodies.

CD4+/CD25+/Foxp3+ regulatory $\mathrm{T}$ cells were analyzed using Foxp3 staining kit (eBioscience, San Diego, CA, USA) according to manufacturer's instructions. $1 \times 10^{6}$ PBMC were incubated with anti-CD4/CD25 cocktail for $30 \mathrm{~min}$. Cells were then washed with cold staining buffer, resuspended in $1 \mathrm{ml}$ fixation/permeabilization buffer and incubated for $30 \mathrm{~min}$. After another washing step and blocking with $2 \%$ normal rat serum, intracellular staining with anti human Foxp3-APC antibody (PCH101) and isotype control $\mathrm{Ab}$ (rat IgG2a) was performed for $30 \mathrm{~min}$. Finally cells were washed with permeabilization buffer, resuspended in staining buffer, and measured as described above. Results are depicted as percent of CD25+Foxp3+ of CD4+ T cells.
Statistical analysis

As detailed above, anti-peptide- and anti-Her-2/neu antibodies were measured by ELISA using six serum dilutions in twofold steps. In simultaneous assays the pre-immunization serum and serum obtained 4 weeks after the third vaccination were measured. Because there is no standard antibody sample against which the obtained OD values can be expressed, a parallel line procedure was chosen and the post-vaccination antibody concentration was expressed as fold-increase above the pre-vaccination serum. The procedure was as follows: in a simultaneous logistic regression analysis all OD values from the pre- and post-vaccination sera were related to the log dilution and a parameter for the time of blood withdrawal (pre- or post-vaccination). The anti-log of the shift from pre- to post-vaccination regression lines is the fold increase of antibody concentrations. Confidence intervals were computed based on Fieller's theorem. The fit of the model was excellent with pseudo- $R^{2}$ values above 0.98 in all cases.

Statistical comparison of Her2/neu peptide vaccinees with the control group of age and sex matched anti-HAV vaccinees was done by matched sample ANOVA with repeated measurements. Differences between groups with respect to the trend from pre- to post-immunization sera were tested based on the interaction source of variance. Fractions of lymphocytes from FACS analyses were arcsine transformed prior to analysis to obtain normality of residuals that was assessed by Lilliefors' corrected Kolmogorov-Smirnov tests. Differences between cytokine concentrations in pre- and post-immunization sera were tested by Wilcoxon's matched-pairs tests. For all analyses a $P$-value below 0.05 was considered significant. No correction for multiple endpoints was applied.

\section{Results}

Recruitment

Ten patients have been recruited. All participants have successfully finished the schedule of three vaccinations.

Evaluation of safety

No local or systemic grade 3 or 4 side effects were observed. Four patients showed a grade 1 local vaccination reaction (Table 2). In particular serological evaluation revealed no signs of hepatic or nephrologic toxicity. With special regard to a possible cardiotoxicity comparable to that seen in the treatment of breast cancer patients with trastuzumab all patients had to undergo measurement of LVEF at screening and at the end of study. No decrease in 
Table 2 Safety evaluation

\begin{tabular}{lll}
\hline $\begin{array}{l}\text { Pat. } \\
\text { number }\end{array}$ & $\begin{array}{l}\text { Local vacc. } \\
\text { reaction grade }\end{array}$ & $\begin{array}{l}\text { Systemic grade } \\
3 / 4 \text { toxicity }\end{array}$ \\
\hline 1 & 1 & No \\
2 & 0 & No \\
3 & 0 & No \\
4 & 1 & No \\
5 & 1 & No \\
6 & 0 & No \\
7 & 0 & No \\
8 & 0 & No \\
9 & 1 & No \\
10 & 0 & No \\
\hline
\end{tabular}

Occurrence of local reactions and systemic side reactions (grades 14) were evaluated

LVEF higher than $10 \%$ was documented in study patients during the study duration.

Course of disease/clinical outcome

One patient died one month after the last vaccination due to disease progression. An extensive evaluation of the course of disease including the last episode of this patient by the investigator as well as by an independent data safety monitoring board (DSMB) did not suggest any vaccinerelated event, but disease progression to be responsible for the patient's death. Another patient died 6 months after the last vaccination with progressive disease while receiving concurrent chemotherapy. At time of study closure (December 2008), the remaining 8 patients were alive, 5 were stable for a period of up to 12 months, 1 showed a partial remission according to RECIST, and 2 had disease progression after 5 and 7 months, respectively.

Immunogenicity of the virosomal-formulated peptide-based vaccine

The vast majority of study participants ( 8 of 10) produced peptide-specific antibodies to all peptides after the 3rd vaccination, as shown in Fig. 1a. Two patients (patient 06 and 10) did not respond to any of the peptides. A moderate titer increase was measured in one patient with existing pre-immunization titer. Baseline levels obtained with serum samples from healthy donors were significantly below the post-immunization titers in the patients (data not shown).

Immunization with Her-2/neu peptides generated Her-2/neu-specific antibodies

Figure $1 \mathrm{~b}$ demonstrates antibody titers specific to the extracellular domain of the Her-2/neu protein determined by ELISA. Her-2/neu-specific IgG, $\kappa$ and/or $\lambda$-chain were detected in 7 of 10 patients after immunization with the multi-peptide vaccine. The ELISA results were verified by Western blot analysis confirming the presence of Her-2/ neu-specific $\operatorname{IgG}$ in 5 of 10 patients. High pre-existing antibody levels against the extracellular domain of Her-2/
Fig. 1 Humoral responses after immunizations with virosomally formulated Her-2/ neu peptides. ELISA analysis was performed with serum samples taken at baseline and 4 weeks after the last immunization. a IgG-specific titers to peptides P4, P6, and P7. b Her-2/neu-specific $\kappa$ - and $\lambda$ light chain IgG. Scatter graphs represents the ratio post-/prevaccination titer. $\mathbf{c}$ Western blot analysis demonstrating increase of Her-2/neu-specific IgG postvaccination in patient 04 . Serum prior (lane 3) and after completed vaccinations (lane 4) was analyzed. Polyclonal rabbit anti Her-2/neu antibody was used as positive control (lane 1), serum of a healthy volunteer as negative control (lane 2)
A
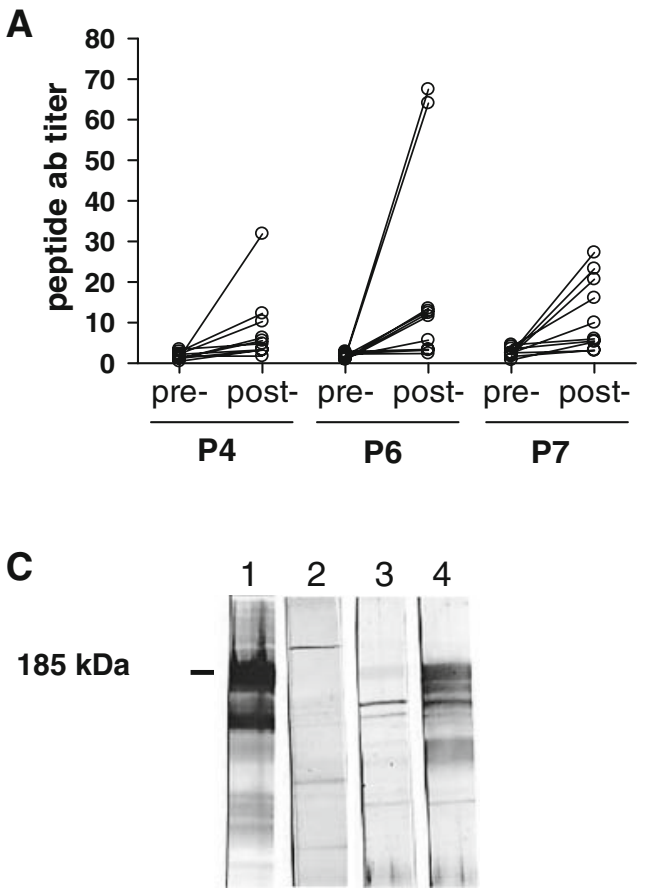

B

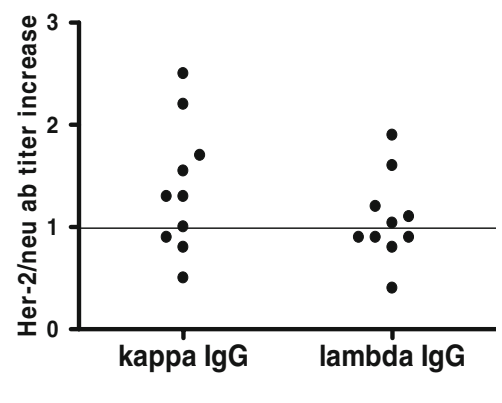


neu was found in one patient; in this case, no increase of Her-2/neu antibody level was achieved during vaccination when measured by ELISA. Qualitative analysis of pre- and post-vaccination serum by Western blot, as shown in Fig. 1c, demonstrates that the Her-2/neu-specific responses to linear epitopes significantly increased after immunizations with peptides.

In general, the $\kappa$-light chain-IgG responses were found in the majority of patients and the magnitude of this isotype was higher than of the $\lambda$-chain-isotype. Interestingly, only $\lambda$-chain light-IgG response was induced in one of the patients (05).

Overall, in 8 of 10 patients induction/increase of Her-2/ neu-specific antibody levels was observed after the threedose vaccination schedule.

\section{Influenza-specific responses/neutralizing antibodies}

Hemagglutination-inhibition test (HI) was performed to measure antibody levels against virosome-associated hemagglutinin. Patients' sera were analyzed at baseline and 4 weeks after the three-dose vaccination schedule. Neutralizing antibodies against A Sing 86 specific-hemagglutinin were detectable in 7 of 10 patients already prior to vaccination. Vaccination with PEV6 elicited a significant HI titer increase ( $\geq 4$-fold) in 6 of 10 patients. In four patients the HI titer was not significantly influenced by the multi-peptide vaccination (Table 3).

Cytokine release after in vitro stimulation with virosomes

Cellular immune responses against the virosomal antigen hemagglutinin were analyzed in PBMC obtained prior and after completion of the vaccination schedule. In 7 of 10

Table 3 Neutralizing antibody titers against influenza associated antigens

\begin{tabular}{lcc}
\hline Patient & Pre-immune & Post-immune \\
\hline 01 & 80 & 160 \\
02 & 40 & 640 \\
03 & 640 & 320 \\
04 & 20 & 160 \\
05 & 20 & 160 \\
06 & 60 & 60 \\
07 & $<10$ & 1280 \\
08 & $<10$ & 160 \\
09 & $<10$ & 160 \\
10 & 80 & 80 \\
\hline
\end{tabular}

HI titers prior and after the three dose vaccination schedule with PEV6 patients an increase of cytokine production was observed after completion of the vaccination schedule. IL-2 production increased significantly $(P=0.023)$, also TNF- $\alpha$ production was markedly higher $(P=0.055)$ as well as IFN- $\gamma$ production (n.s.). In one patient, high cytokine levels already existed in cultures prior to vaccination and remained at similar level after the third vaccination. In cell cultures of another patient, cytokine concentrations before and after vaccination were below detection limit. A decrease of pre-vaccination cytokine secretion was observed in PBMC of one patient. Of note, this patient displayed also insufficient humoral responses to peptides and influenza antigens (Fig. 2).

Analysis of surface marker expression on lymphocytes

We assessed various subpopulations of PBMC in all study patients prior and after completion of vaccinations as well as in eight age- and gender-matched healthy volunteers. Cell surface marker expression on B cells (CD19), memory B cells (CD19/CD27), T cells (CD4, CD8), memory subpopulations (CD45R0), as well as CD25 (IL-2 receptor $\alpha$ chain), and L-selectin (CD62L) expression on CD4 lymphocytes were evaluated (Table 4). A significant decrease of CD3 T lymphocytes $(P=0.017)$ compared to healthy controls was noticed after completion of the vaccination series. The B cell lymphocyte population tended to increase in the study group post-vaccination, similarly as in the healthy donor group. CD4+ and CD8+ T cells did, however, not significantly differ before and after vaccination between the study patients and the healthy donor group. The L-selectin (CD62L) expressing subpopulation declined in 6 of 10 study patients after vaccination (Table 4).

The median value of $\mathrm{CD} 4+/ \mathrm{CD} 25+$ Foxp3+ regulatory $\mathrm{T}$ cells in circulating PBMC of the breast cancer patients prior to vaccination was 4.86 [3.18-6.54] and significantly declined after vaccination to 4.02 [2.74-5.95], $P=0.007$ (Fig. 3). In the healthy control group the median values of CD4+CD25+Foxp3+ T reg cells prior (3.98 [2.90-3.66]) and after vaccination (2.69 [2.63-3.10]) were significantly $(P<0.033)$ lower than in the tumor patients (Fig. 3).

\section{Discussion}

Several studies in experimental models including our own preclinical data [8, 9] have demonstrated that successful reduction of Her-2/neu overexpressing tumors after vaccination requires both humoral and cellular immune responses. Here, we demonstrate the results of a single center phase I clinical trial showing that a Her-2/neu multipeptide vaccine is safe and immunogenic concerning $\mathrm{B}$ - 

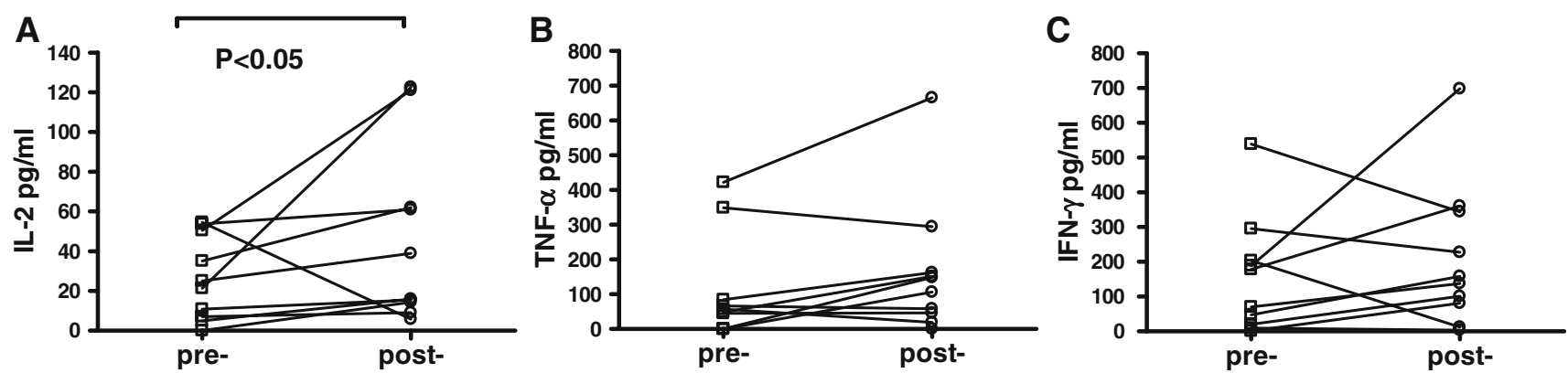

Fig. 2 Cellular responses to influenza antigens in vitro. PBMC isolated at baseline and 4 weeks after the last vaccination were stimulated with virosomes containing hemagglutinin. Cytokine production in supernatants was measured by ELISA. Data represent values after subtraction of unstimulated background

Table 4 Characterization of PBMC by surface marker expression analyzed by flow cytometry

\begin{tabular}{|c|c|c|c|c|c|c|c|}
\hline \multirow[t]{2}{*}{ Cell type } & \multirow{2}{*}{$\begin{array}{l}\mathrm{CD} \\
\text { marker }\end{array}$} & \multicolumn{2}{|c|}{ Study patients $n=10$} & \multicolumn{2}{|c|}{ Normal donors $n=8$} & \multirow{2}{*}{$\begin{array}{l}\text { Pre-values* } \\
P\end{array}$} & \multirow{2}{*}{$\begin{array}{l}\text { Trend } \\
P\end{array}$} \\
\hline & & Pre- & Post- & Pre- & Post- & & \\
\hline T lymphocytes & CD3 & $66.5[60.2-73.3]$ & $62.1[54.9-64.4]$ & $73.6[63.4-75.9]$ & $73.6[66.5-78.4]$ & 0.332 & 0.017 \\
\hline B lymphocytes & CD19 & $7.9[5.9-12.5]$ & $12.4[10-14.5]$ & 8.9 [6.6-14.8] & $9.3[8.2-13.1]$ & 0.643 & 0.100 \\
\hline \multirow[t]{4}{*}{$\mathrm{T}$ cells/memory } & $\mathrm{CD} 4$ & $44.2[38.7-52.3]$ & $43.0[37.0-46.0]$ & $53.8[38.5-57.0]$ & $49.1[37.0-55.3]$ & 0.678 & 0.667 \\
\hline & CD4/CD45R0 & $36.6[30.6-41.6]$ & $32.9[27.4-37.6]$ & 31.1 [29.3-32.9] & $31.0[28.0-32.0]$ & 0.102 & 0.367 \\
\hline & CD8 & $20.5[18.6-26.0]$ & $21.3[18.2-24.5]$ & $21.8[17.8-31.3]$ & $25.3[17.8-28.2]$ & 0.822 & 0.360 \\
\hline & CD8/CD45R0 & $11.5[10.2-13.0]$ & $10.3[8.1-10.7]$ & $9.2[6.8-15.7]$ & $12.6[6.8-16.0]$ & 0.980 & 0.075 \\
\hline $\begin{array}{l}\text { Peripheral lymph } \\
\text { node homing } \\
\text { receptor }\end{array}$ & $\mathrm{CD} 4 / \mathrm{CD} 62 \mathrm{~L}$ & $16.7[6.4-22.9]$ & $8.9[5.0-14.6]$ & $13.9[11.5-27.6]$ & $16.5[12.8-22.3]$ & 0.757 & 0.265 \\
\hline $\begin{array}{l}\text { High affinity } \\
\text { IL-2 receptor }\end{array}$ & $\mathrm{CD} 4 / \mathrm{CD} 25$ & $17.3[11.3-21.4]$ & $15.6[7.7-19.3]$ & $16.5[10.7-21.5]$ & $13.7[10.2-23.3]$ & 0.897 & 0.198 \\
\hline
\end{tabular}

* $P$-values for comparison of preimmune cell populations in patients and normal donors

* $P$-values for comparison of trend (pre-immune-post-immune cell populations) between patients and normal donors

Fig. 3 a Frequency of regulatory $\mathrm{T}$ cells assessed by flow cytometry in tumor patients and healthy controls. Data depict percentage of CD4+ T cells expressing CD25 and Foxp3 before and after vaccinations with the Her-2/neu peptide vaccine in tumor patients as well as in healthy age- and gender-matched donors prior and after vaccination with a hepatitis A vaccine. b Scatter plots of two representative tumor patients and one healthy control. Cells were gated on the CD4+ population of lymphocytes, the numbers represent percent of each quadrant
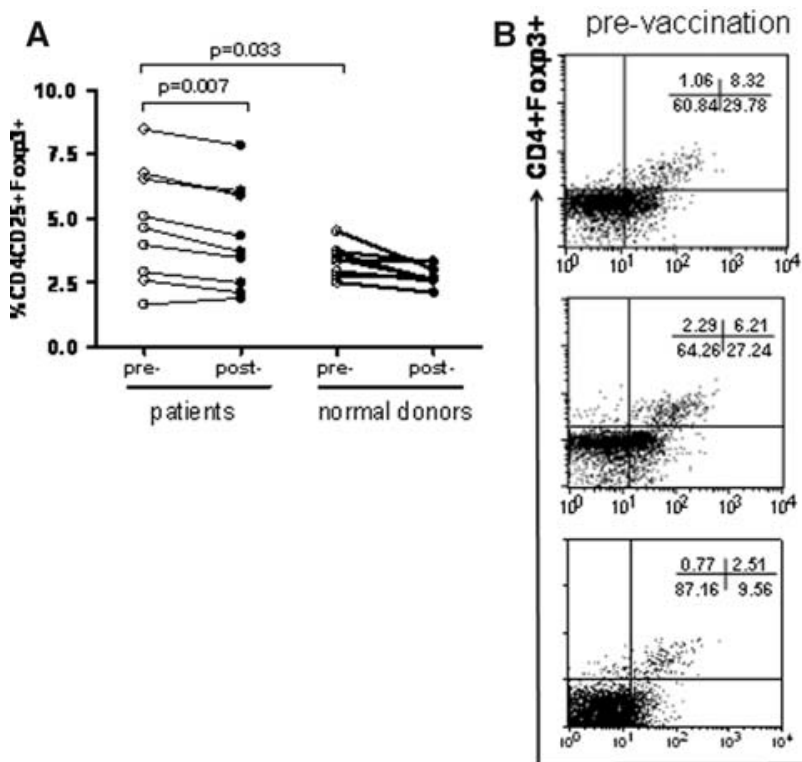

post-vaccination
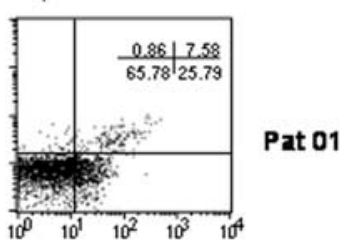

01

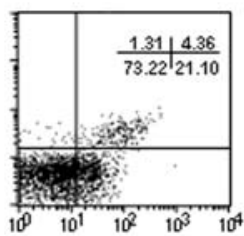

Pat 02

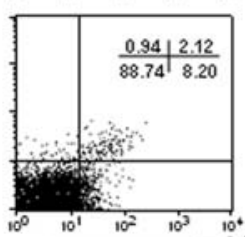

donor 
and $\mathrm{T}$ cell responses in patients with advanced breast cancer. The current protocol foresaw vaccination in patients with metastatic breast cancer without overexpression of the Her-2/neu protein $(+$ and ++ upon immunohistochemistry, FISH negative), which was due to ethical reasons in order not to withhold standard anti-Her-2/neu immunotherapy with trastuzumab from patients with Her2/neu-driven disease.

According to the current trial conduct, this phase I study had safety as primary endpoint. Additionally, immunogenicity of the vaccine was chosen as secondary endpoint, since the specificity of the evoked immune responses toward Her-2/neu is of utmost importance as previously demonstrated in our preclinical studies. Characterization of the B- and T cell compartment was particularly interesting, as immune responsiveness has been repeatedly shown to be perturbed in cancer patients [15-17].

Unlike many peptide vaccines against cancers which have been designed to induce cytotoxic $\mathrm{T}$ cell responses using class I restricted peptides [18-23], we used putative B cell epitopes from the extracellular domain of the Her-2/ neu protein being HLA type independent. In order to induce $\mathrm{CD} 4+\mathrm{T}$ helper cell responses, important for the induction of antibody isotype switching and memory responses [24], these peptides were coupled to immunopotentiating reconstituted influenza virosomes (IRIV), an adjuvant system currently used in two licensed vaccines, a hepatitis A vaccine and an influenza vaccine, which proved to cause minimal side effects while being very immunogenic $[11,25]$.

Indeed, the results from the primary study endpoint on vaccine safety showed that only 4 of 10 vaccinees displayed minimal local reactions of grade 1 , consisting of mild redness and swelling or pain at the injection site, while no fever or other systemic side effects (grade 4/5) were reported. The deaths of two study participants, 1 and 6 months after vaccination, respectively, were not vaccinerelated but due to disease progression. On these occasions an independent DSMB arrived at the same conclusion as the investigators. Half of the patient collective $(50 \%)$ presented with stable disease up to 12 months after vaccination. Six months after initiation of vaccination one patient showed a partial remission according to RECIST and three developed tumor progression (Table 2). As all patients received hormone treatment and did not show Her$2 /$ neu overexpression it is more probable to attribute any clinical effects to the applied endocrine medication rather than to the Her-2/neu vaccine.

The second endpoint of the study concentrated on the immunogenicity of the vaccine. The majority of patients, 8 of 10 , developed a significant increase in anti-peptide titers to all three peptides (Fig. 1a), while only two participants did not respond with peptide-specific titers. As all patients received endocrine therapy by aromatase inhibitors during this vaccination trial for ethical reasons, the question whether aromatase inhibition might have influenced the magnitude of the antibody responses remains unanswered to date. This appears to be unlikely though, because recent studies with other adjuvant therapies have shown not to impact on immune response levels [26].

Importantly, in all but one of the peptide-responsive vaccinees, the antibody levels were also directed against the native Her-2/neu antigen. The Her-2/neu antibody responses were measured by quantitative ELISA and verified by Western blot analysis. The discrepancy in the detected number of Her-2/neu-specific IgG between the two methods might be explained by the fact that only linear epitopes can be recognized by Western blot analysis, while also conformational epitopes can be measured by ELISA. In contrast to other studies [27], the results from our trial indicate that the B cell-derived peptide vaccine was potent enough to overcome tolerance to the self-antigen Her-2/neu (Fig. 1b, c). Whether the quality of the induced antibodies is related to the kappa or lambda light chain $\operatorname{IgG}$ is impossible to estimate at this stage. Studies on the antibody avidity, as described by Goldblatt et al. [28], could give further indications on the biological activity of the induced antibodies. It must be stressed, however, that this phase I study does not allow any evaluation of antibody/vaccine efficacy, since the selection of patients with only weak Her-2/neu expression, due to ethical reasons in order not to withhold trastuzumab treatment when indicated, does not represent the actual target population in which the antitumor effects of the induced antibodies can be properly evaluated. Along with further assessment of safety, evaluation of efficacy of the present vaccine would be the focus of a subsequent phase II trial.

It has been proposed that successful vaccination to generate Her-2/neu immunity is not only dependent on the appropriate selection of epitopes but also on the use of a potent adjuvant system. In this respect, we chose IRIV as conjugation partner for two reasons: firstly, the presence of the influenza hemagglutinin antigen from the influenza A Singapore strain on the virosome surface to which the majority of the population has previously been exposed allows the induction of booster responses to this antigen. Accordingly, the majority of the vaccinees displayed a significant increase in the influenza neutralizing antibody titers (Table 3). The two mentioned Her-2/neu nonresponder vaccinees also failed to develop influenza-specific antibody levels indicative for immunological incompetence. Secondly, the virosome system was used to generate bystander CD4+ T cell responses [24] necessary to induce antibody isotype switching to $\mathrm{IgG}$ as well as memory responses to the $\mathrm{B}$ cell peptides. The production of IL-2 in particular, but also of TNF- $\alpha$ as well as IFN- $\gamma$ 
markedly increased in cultures of virosome-stimulated PBMCs after completion of the vaccination series. These results are in line with our preclinical data showing that the selected B cell peptides from the ECD in conjunction with a strong adjuvant system were able to induce both, humoral and cellular responses. Noteworthy, the vaccine was immunogenic (except for two) in patients across all HLA types in stage IV disease. Thus, our study indicates that patients with advanced metastatic breast cancer are immunocompetent and fully susceptible to vaccination. This is further supported by characterization of the lymphocyte population based on CD surface marker expression in comparison to age- and gender-matched healthy controls vaccinated with a hepatitis A vaccine [13] (Table 4). Except for the number of $\mathrm{CD} 3+\mathrm{T}$ cells, no significant differences in the distribution of lymphocyte subpopulations (CD4, CD45RO, CD8, CD19) were detected between the tumor patients and the healthy controls before and after vaccination. Also the percentage of $\mathrm{CD} 4 \mathrm{~T}$ cells expressing CD62L, a homing receptor on naïve $\mathrm{T}$ cells, did not significantly differ between the two groups and declined after vaccination, most probably as a result of immune responsiveness to the vaccine antigens, as also described for other vaccines in healthy adults [13, 29].

Several studies have shown that $\mathrm{T}$ regulatory cells (Tregs) are increased in patients with a variety of malignancies and that higher numbers of Tregs are associated with tumor progression [30-34]. Recently, it was shown that tumor infiltration by Tregs is associated with a reduced survival of breast cancer patients [35]. Treg cells may also be responsible for the failure of an anticancer immune response by hindering the generation and activity of antitumor reactive $\mathrm{T}$ cells thereby maintaining self-tolerance to the tumor antigens. Along these lines, also our data showed that the patients had a significantly higher number of circulating Treg cells, defined as CD4+CD25+Foxp3+ T cells, than the healthy matched control group prior to vaccination. Of notice was the fact that the number of Tregs significantly decreased after vaccination with the multi-peptide vaccine (Fig. 3). The fact that a decline in Treg cells was also observed in healthy donors after vaccination with a hepatitis A vaccine (Fig. 3) raises the question whether each vaccination has the potential to interfere with this cell population in order to guarantee optimal immune responses. In tumor patients this suppressive effect on Tregs might be particularly crucial for the efficacy of a vaccine. Similar results have very recently been described in breast cancer patients treated with trastuzumab [30] as well as with an aromatase inhibitor [35], thus suggesting that overcoming Treg cell reconstitution might not only be seen in the present context, but perhaps also as an important contributor to success in a wider range of anti-tumor treatment strategies.
Taken together, the results from our phase I trial show that the described Her-2/neu vaccine was safe and immunogenic in 8 of 10 patients. As a next step it is of interest whether an increased vaccine dose might lead to an ameliorated seroconversion rate. Current studies on the impact of higher peptide vaccine doses on anti-tumor immunity show controversial results [36, 37]. Thus, a second part of this phase I study is on the way to be able to answer this question. In future settings, a series of different options for further phase II studies exist, such as the combination of the current vaccine with passive antibody administration or with other treatment modalities including Treg cell inhibitors.

Acknowledgments The study was supported by a grant from BioLife Science and by the Medical University of Vienna. The assistance of Mrs Marika Rosner as study nurse during the phase I clinical trial is very much appreciated.

\section{References}

1. Slamon DJ, Clark GM, Wong SG, Levin WJ, Ullrich A, McGuire WL (1987) Human breast cancer: correlation of relapse and survival with amplification of the HER-2/neu oncogene. Science 235(4785): 177-182

2. Andrulis IL, Bull SB, Blackstein ME et al (1998) neu/erbB-2 amplification identifies a poor-prognosis group of women with node-negative breast cancer. Toronto Breast Cancer Study Group. J Clin Oncol 16(4):1340-1349

3. Suter TM, Procter M, van Veldhuisen DJ et al (2007) Trastuzumab-associated cardiac adverse effects in the herceptin adjuvant trial. J Clin Oncol 25(25):3859-3865

4. Maloney DG, Grillo-Lopez AJ, Bodkin DJ et al (1997) IDECC2B8: results of a phase I multiple-dose trial in patients with relapsed non-Hodgkin's lymphoma. J Clin Oncol 15(10):32663274

5. Vogel CL, Cobleigh MA, Tripathy D et al (2002) Efficacy and safety of trastuzumab as a single agent in first-line treatment of HER2-overexpressing metastatic breast cancer. J Clin Oncol 20(3):719-726

6. Rosenberg SA, Yang JC, Restifo NP (2004) Cancer immunotherapy: moving beyond current vaccines. Nat Med 10(9):909915

7. Lollini PL, Cavallo F, Nanni P, Forni G (2006) Vaccines for tumour prevention. Nat Rev Cancer 6(3):204-216

8. Jasinska J, Wagner S, Radauer C et al (2003) Inhibition of tumor cell growth by antibodies induced after vaccination with peptides derived from the extracellular domain of Her-2/neu. Int J Cancer 107(6):976-983

9. Wagner S, Jasinska J, Breiteneder H, Kundi M, Pehamberger H, Scheiner O, Zielinski CC, Wiedermann U (2007) Delayed tumor onset and reduced tumor growth progression after immunization with a Her-2/neu multi-peptide vaccine and IL-12 in c-neu transgenic mice. Breast Cancer Res Treat 106(1):29-38

10. Zurbriggen $R$ (2003) Immunostimulating reconstituted influenza virosomes. Vaccine 21(9-10):921-924

11. Bovier PA (2008) Epaxal: a virosomal vaccine to prevent hepatitis A infection. Expert Rev Vaccines 7(8):1141-1150

12. Thompson FM, Porter DW, Okitsu SL et al (2008) Evidence of blood stage efficacy with a virosomal malaria vaccine in a phase IIa clinical trial. PLoS ONE 3(1):e1493 
13. Garner-Spitzer E, Kundi M, Rendi-Wagner P et al (2009) Correlation between humoral and cellular immune responses and the expression of the hepatitis A receptor HAVcr-1 on T cells after hepatitis A re-vaccination in high and low-responder vaccinees. Vaccine 27(2):197-204

14. Phillips CA, Forsyth BR, Christmas WA, Gump DW, Whorton EB, Rogers I, Rudin A (1970) Purified influenza vaccine: clinical and serologic responses to varying doses and different routes of immunization. J Infect Dis 122(1):26-32

15. Zielinski CC, Stuller I, Dorner F, Potzi P, Muller C, Eibl MM (1986) Impaired primary, but not secondary, immune response in breast cancer patients under adjuvant chemotherapy. Cancer 58(8):1648-1652

16. Molling JW, Kolgen W, van der Vliet HJ et al (2005) Peripheral blood IFN-gamma-secreting Valpha24+Vbeta11+NKT cell numbers are decreased in cancer patients independent of tumor type or tumor load. Int J Cancer 116(1):87-93

17. Liyanage UK, Moore TT, Joo HG et al (2002) Prevalence of regulatory $\mathrm{T}$ cells is increased in peripheral blood and tumor microenvironment of patients with pancreas or breast adenocarcinoma. J Immunol 169(5):2756-2761

18. Fisk B, Blevins TL, Wharton JT, Ioannides CG (1995) Identification of an immunodominant peptide of HER-2/neu protooncogene recognized by ovarian tumor-specific cytotoxic $\mathrm{T}$ lymphocyte lines. J Exp Med 181(6):2109-2117

19. Disis ML, Grabstein KH, Sleath PR, Cheever MA (1999) Generation of immunity to the HER-2/neu oncogenic protein in patients with breast and ovarian cancer using a peptide-based vaccine. Clin Cancer Res 5(6):1289-1297

20. Disis ML, Gralow JR, Bernhard H, Hand SL, Rubin WD, Cheever MA (1996) Peptide-based, but not whole protein, vaccines elicit immunity to HER-2/neu, oncogenic self-protein. J Immunol 156(9):3151-3158

21. Matsui S, Ahlers JD, Vortmeyer AO, Terabe M, Tsukui T, Carbone DP, Liotta LA, Berzofsky JA (1999) A model for CD8+ CTL tumor immunosurveillance and regulation of tumor escape by CD4 T cells through an effect on quality of CTL. J Immunol 163(1):184-193

22. Welters MJ, Kenter GG, Piersma SJ et al (2008) Induction of tumor-specific CD4+ and CD8+ T-cell immunity in cervical cancer patients by a human papillomavirus type 16 E6 and E7 long peptides vaccine. Clin Cancer Res 14(1):178-187

23. Peoples GE, Holmes JP, Hueman MT et al (2008) Combined clinical trial results of a HER2/neu (E75) vaccine for the prevention of recurrence in high-risk breast cancer patients: U.S. Military Cancer Institute Clinical Trials Group Study I-01 and I02. Clin Cancer Res 14(3):797-803

24. Hung K, Hayashi R, Lafond-Walker A, Lowenstein C, Pardoll D, Levitsky H (1998) The central role of CD4(+) T cells in the antitumor immune response. J Exp Med 188(12):2357-2368
25. Kunzi V, Dornseiff M, Horwath J, Hartmann K (2009) Safe vaccination of children with a virosomal adjuvanted influenza vaccine. Vaccine 27(8):1261-1265

26. Coveler AL, Goodell V, Webster DJ, Salazar LG, Fintak PA, Childs JS, Higgins DM, Disis ML (2009) Common adjuvant breast cancer therapies do not inhibit cancer vaccine induced $\mathrm{T}$ cell immunity. Breast Cancer Res Treat 113(1):95-100

27. Disis ML, Goodell V, Schiffman K, Knutson KL (2004) Humoral epitope-spreading following immunization with a HER-2/neu peptide based vaccine in cancer patients. J Clin Immunol 24(5):571-578

28. Goldblatt D, Vaz AR, Miller E (1998) Antibody avidity as a surrogate marker of successful priming by Haemophilus influenzae type $\mathrm{b}$ conjugate vaccines following infant immunization. $\mathrm{J}$ Infect Dis 177(4):1112-1115

29. Pfister G, Weiskopf D, Lazuardi L et al (2006) Naive T cells in the elderly: are they still there? Ann N Y Acad Sci 1067:152-157

30. Horlock C, Stott B, Dyson PJ, Morishita M, Coombes RC, Savage P, Stebbing J (2009) The effects of trastuzumab on the CD4+CD25+FoxP3+ and CD4+IL17A+ T-cell axis in patients with breast cancer. Br J Cancer 100(7):1061-1067

31. Mizukami Y, Kono K, Kawaguchi Y, Akaike H, Kamimura K, Sugai H, Fujii H (2008) Localisation pattern of Foxp3+ regulatory $\mathrm{T}$ cells is associated with clinical behaviour in gastric cancer. Br J Cancer 98(1):148-153

32. Kono K, Kawaida H, Takahashi A, Sugai H, Mimura K, Miyagawa N, Omata H, Fujii H (2006) CD4(+)CD25 high regulatory T cells increase with tumor stage in patients with gastric and esophageal cancers. Cancer Immunol Immunother 55(9):1064-1071

33. Vence L, Palucka AK, Fay JW, Ito T, Liu YJ, Banchereau J, Ueno $H$ (2007) Circulating tumor antigen-specific regulatory $T$ cells in patients with metastatic melanoma. Proc Natl Acad Sci USA 104(52):20884-20889

34. Enarsson K, Lundgren A, Kindlund B, Hermansson M, Roncador G, Banham AH, Lundin BS, Quiding-Jarbrink M (2006) Function and recruitment of mucosal regulatory $\mathrm{T}$ cells in human chronic Helicobacter pylori infection and gastric adenocarcinoma. Clin Immunol 121(3):358-368

35. Generali D, Bates G, Berruti A et al (2009) Immunomodulation of FOXP3 + regulatory $\mathrm{T}$ cells by the aromatase inhibitor letrozole in breast cancer patients. Clin Cancer Res 15(3):1046-1051

36. Disis ML, Schiffman K, Guthrie K, Salazar LG, Knutson KL, Goodell V, dela Rosa C, Cheever MA (2004) Effect of dose on immune response in patients vaccinated with an her-2/neu intracellular domain protein-based vaccine. J Clin Oncol 22(10): 1916-1925

37. Holmes JP, Gates JD, Benavides LC et al (2008) Optimal dose and schedule of an HER-2/neu (E75) peptide vaccine to prevent breast cancer recurrence: from US Military Cancer Institute Clinical Trials Group Study I-01 and I-02. Cancer 113(7):1666-1675 